Dr Harbinson's final comment about my victimising other psychiatrists is rather emotive. He/she is correct regarding the speculative nature of some aspects of my paper. This has been necessary because of the relative lack of research. However, I am certainly not intending that criticism be directed at colleagues, a number of whom have carried out valuable research while also carrying heavy clinical workloads. It remains my view that there is a need for a detailed epidemiological community study which specifically addresses the issue of trauma.

Curran, P. S. (1988) Psychiatric aspects of terrorist violence; Northern Ireland 1969-1987. British Journal of Psychiatry, 153, 470-475.

Department of Health and Social Services (1998) Living with the Trauma of the Troubles. Belfast: Social Services Inspectorate, DHSS (NI).

Lyons, H. A. (197I) Psychiatric sequelae of the Belfast riots. British Journal of Psychiatry, II8, 265-273.

Pollock, P. H. (1999) When the killer suffers: posttraumatic stress reactions following homicide. Legal and Criminological Psychology, 4, 185-202.

Smyth, M. (1998) Half the Battle: Understanding the Effects of the 'Troubles' on Children and Younger People in Northern Ireland. Belfast: INCORE.

O. E. Daly Department of Psychiatry, Lagan Valley Hospital, Hillsborough Road, Lisburn, BT28 IJP

\section{Studying grief in adults with learning disabilities}

I read with interest the article by BonellPascual et al (1999) and would agree that recognition of, and service provision for, the bereaved adult learning disability population is markedly deficient. However, their conclusion that learning disability is a significant predictor of future mental health problems following bereavement is not substantiated and should be interpreted with caution in view of the flawed research methodology.

The original cohort reported short-term psychopathological and behavioural outcomes following bereavement (Hollins \& Esterhuyzen, 1997). This case-controlled study found an increase in aberrant behaviours and psychopathology in those bereaved compared with controls. BonellPascual et al aimed to investigate whether the same outcomes had changed over a longer term (six to eight years). Unfortunately, the control cohort was not followed up, thereby making meaningful inferences impossible, as confounding life events are not controlled for. Also, more than half the study population had additional medical disorders of likely prognostic significance. The authors recognise the limitations of their small sample size, but this is further compounded by incomplete follow-up of the original cohort, with greater than $15 \%$ of the original cohort excluded. Furthermore, follow-up data are missing from the analysis of psychopathology and aberrant behaviour.

In the classification of psychopathology, each sub-scale shows varied changes with no interpretable trends over time. Psychopathology identified in the original 1997 study had resolved in over $70 \%$, although a few new cases were identified, especially in the adjustment and anxiety disorder sub-categories. However, we cannot attribute these new cases to underlying learning disability as confounding medical and life events may have played a significant role. Aberrant behaviour patterns were reported as showing a mean increase in each sub-scale over time. The individual change in aberrant behaviour patterns was, however, in both directions in all sub-scales (i.e. an improvement and deterioration in behaviour). The mean change quoted by the authors in such a small study sample with wide ranges of behaviour is, therefore, unreliable. Similarly, the one statistically 'significant' result, showing inappropriate speech to be more common, should also be treated with caution and taken in isolation is unlikely to be of clinical significance. Furthermore, the authors allude to problems with validation of the behavioural and psychopathological measures used in the significant proportion of the study population who were taking antipsychotic and antidepressant medication.

It is now recognised that people with learning disability do understand the permanence of death and consequently grieve (Harper \& Wadsworth, 1993; Read, 1996). Bonell-Pascual et al highlight the need to better understand the general and specific needs of this vulnerable group of people and provide some useful insight into effective interventions. Future studies should be aimed at addressing these issues, be carried out prospectively and have adequate control groups to allow safe and meaningful conclusions to be drawn.

Bonell-Pascual, E., Huline-Dickens, S., Hollins, S., et al (1999) Bereavement and grief in adults with learning disabilities. A follow-up study. British Journal of Psychiatry, I75, 348-350.

Harper, D. C. \& Wadsworth, J. S. (1993) Grief in adults with mental retardation: preliminary findings. Research in Developmental Disabilities, 14, 313-330.
Hollins, S. \& Esterhuyzen, A. (1997) Bereavement and grief in adults with learning disabilities. British Journal of Psychiatry, I70, 497-50I.

Read, S. (1996) Helping people with learning disabilities to grieve. British Journal of Nursing, 5, 91-95.

C. A. Lyons Department of Psychiatry of Learning Disability, Black Country Mental Health Trust, Heath Lane Hospital, West Bromwich

Author's reply: We have read Dr Lyons' letter with interest, and considered his/her comments with great care. It is encouraging that the letter also highlights the need for further research in this area, and we are happy to say that we have new research in progress.

We acknowledge that the methodology of the published report has limitations, but that does not mean that it is flawed. Dr Lyons does little more than amplify the limitations spelled out in what was, after all, only a short research report. It is perfectly acceptable to use data from the first study as longitudinal data in the follow-up study. Furthermore, although follow-up of the original control group might have yielded some useful material, it is more probable that it would have been unreliable, because of the likelihood that these individuals would have experienced bereavements themselves in the intervening time between the first and second study.

Dr Lyons suggests that new 'cases' at follow up cannot be attributed to the underlying learning disability as confounding medical and life events may have played a part. One of the key points made in both 1997 and 1999 papers is that the effects of bereavement are compounded by the increase in life events experienced by the client group at such a time.

With regard to the size of the second sample, the shortfall is not excessive: as stated in the paper, three of the missing individuals were dead, and three untraceable, possibly also dead. The true followup rate could thus be more accurately described as 41 out of 44 , or $93 \%$. Furthermore, two of the remaining three carers refused to help with the follow-up interviews because bereavement was too sensitive an issue for either the relative or the person with learning disability.

Dr Lyons suggests that the results of the study are not 'meaningful'. The original manuscript, which was cut in length at the request of the Editor, included qualitative material collected from carers at the same time as the quantitative data. This material supports the results of the quantitative data, 